\title{
Density-matrix-power functional: Performance for finite systems and the homogeneous electron gas
}

\author{
N. N. Lathiotakis, ${ }^{1,2,3}$ S. Sharma, ${ }^{4,2,3}$ J. K. Dewhurst, ${ }^{5}$ F. G. Eich, ${ }^{4,2,3}$ M. A. L. Marques, ${ }^{6,7,3}$ and E. K. U. Gross ${ }^{2,3}$ \\ ${ }^{1}$ Theoretical and Physical Chemistry Institute, NHRF, Vas. Constantinou 48, GR-11635 Athens, Greece \\ ${ }^{2}$ Institut für Theoretische Physik, Freie Universität Berlin, Arnimallee 14, D-14195 Berlin, Germany \\ ${ }^{3}$ European Theoretical Spectroscopy Facility \\ ${ }^{4}$ Fritz Haber Institute of the Max Planck Society, Faradayweg 4-6, D-14195 Berlin, Germany \\ ${ }^{5}$ School of Chemistry, The University of Edinburgh, Edinburgh EH9 3JJ, United Kingdom \\ ${ }^{6}$ Laboratoire de Physique de la Matière Condensée et Nanostructures, Université Lyon I, CNRS, UMR 5586, \\ Domaine Scientifique de la Doua, F-69622 Villeurbanne Cedex, France \\ ${ }^{7}$ Department of Physics, Centre for Computational Physics, University of Coimbra, 3004-516 Coimbra, Portugal
}

(Received 24 December 2008; published 16 April 2009)

\begin{abstract}
An exchange-correlation energy functional involving fractional power of the one-body reduced density matrix [S. Sharma, J. K. Dewhurst, N. N. Lathiotakis, and E. K. U. Gross, Phys. Rev. B 78, 201103(R) (2008)] is applied to finite systems and to the homogeneous electron gas. The performance of the functional is assessed for the correlation and atomization energies of a large set of molecules and for the correlation energy of the homogeneous electron gas. High accuracy is found for these two very different types of systems.
\end{abstract}

DOI: 10.1103/PhysRevA.79.040501

PACS number(s): 31.10.+z, 31.15.A-, 31.15.ve, 31.15.vq

For the past 40 years, density-functional theory (DFT) developed into one of the most successful theories in the study of the electronic structure of atoms, molecules, and periodic solids. At its heart lies the exchange-correlation (xc) functional, for which many approximations have been proposed. The simplest functionals that depend only on the density [the local-density approximation (LDA)], or on the density and its gradients [the generalized gradient approximation (GGA)], give a very satisfactory description of many ground-state properties. However, they still fail to reach chemical accuracy for some important quantities such as reaction or atomization energy. To remedy this situation hybrid functionals were introduced, the first and most widely used example being the Becke three-parameter Lee-Yang-Parr (B3LYP) functional $[1,2]$. This functional is able to reproduce experimental atomization energies within about $10 \%$ error. Although the atomization energies obtained using B3LYP are in good agreement with experiments, the absolute correlation energies, an accurate description of which can be thought of as a test for the quality of any approximate functional, exhibit a sizeable error (up to 400\%) [3]. This is not a surprise since experimentally one normally measures energy differences, and it is these quantities that functionals such as B3LYP are designed to reproduce. Accurate correlation energies for finite systems can be obtained by going beyond the DFT framework, for instance, by using Møller-Plesset second-order (MP2) perturbation theory or the coupled cluster method with singles, doubles, and perturbative triples $[\operatorname{CCSD}(\mathrm{T})]$. However, these methods are computationally too expensive to be applied to realistic systems of ever growing complexity: biomolecules, large clusters, and nanodevices to name but a few examples.

Recently, reduced density-matrix-functional theory (RDMFT) has appeared as an alternative approach to handle complex systems. It has shown great potential for improving upon DFT results for finite systems. RDMFT uses the onebody reduced density matrix (1-RDM) $\gamma$ as the basic variable
[4,5]. This quantity, for the ground state, is determined through the minimization of the total energy functional under the constraint that $\gamma$ is ensemble $N$ representable. The total energy as a functional of $\gamma$ can be expressed as (atomic units are used throughout)

$$
\begin{aligned}
E_{v}[\gamma]= & \int d^{3} r \int d^{3} r^{\prime} \delta\left(\mathbf{r}-\mathbf{r}^{\prime}\right)\left[-\frac{\nabla^{2}}{2}\right] \gamma\left(\mathbf{r}, \mathbf{r}^{\prime}\right) \\
& +\int d^{3} r v(\mathbf{r}) \rho(\mathbf{r}) \\
& +\frac{1}{2} \int d^{3} r \int d^{3} r^{\prime} \frac{\rho(\mathbf{r}) \rho\left(\mathbf{r}^{\prime}\right)}{\left|\mathbf{r}-\mathbf{r}^{\prime}\right|}+E_{\mathrm{xc}}[\gamma],
\end{aligned}
$$

where $\rho(\mathbf{r})$ (the electron density) is the diagonal of the $1-\mathrm{RDM}$ and $v(\mathbf{r})$ is the external potential. The first two terms in Eq. (1) are the kinetic and external potential energies. The electron-electron interaction can be cast in the last two terms, the first being the Coulomb repulsion energy and $E_{\mathrm{xc}}$ the xc energy functional. In principle, Gilbert's [5] generalization of the Hohenberg-Kohn theorem to the 1-RDM guarantees the existence of a functional $E_{v}[\gamma]$ whose minimum yields the exact $\gamma$ and the exact ground-state energy of the systems characterized by the external potential $v(\mathbf{r})$. In practice, however, the xc energy is an unknown functional of the 1-RDM and needs to be approximated. In the past years, a plethora of approximate functionals has been introduced. [6-15] An assessment of the relative performance of these functionals for a large set of atoms and molecules reveals that the so-called third correction to Buijse Baerends functional (BBC3) [13] and Piris natural orbital functional (PNOF0) $[14,3]$ functionals yield results for molecular systems, with an accuracy comparable to the MP2 method [3,14,16-19].

The situation for extended systems is somewhat more complicated. When applied to the simplest system, the homogeneous electron gas (HEG), these functionals lead to 
rather inaccurate correlation energies [11]. Moreover, for periodic solids, the aforementioned functionals fail to reproduce the fundamental gaps for insulators [20] (band as well as Mott). A functional was presented by Sharma et al. [20] to solve this problem. It reads

$$
E_{\mathrm{xc}}[\gamma]=-\frac{1}{2} \int d^{3} r \int d^{3} r^{\prime} \frac{\left|\gamma^{\alpha}\left(\mathbf{r}, \mathbf{r}^{\prime}\right)\right|^{2}}{\left|\mathbf{r}-\mathbf{r}^{\prime}\right|},
$$

where $\gamma^{\alpha}$ indicates the power used in the operator sense, i.e.,

$$
\gamma^{\alpha}\left(\mathbf{r}, \mathbf{r}^{\prime}\right)=\sum_{j}\left(n_{j}\right)^{\alpha} \phi_{j}^{*}\left(\mathbf{r}^{\prime}\right) \phi_{j}(\mathbf{r}) .
$$

Here $\phi_{j}(\mathbf{r})$ denotes the natural orbitals and $n_{j}$ denotes their occupation numbers. The functional in Eq. (2) was shown to perform very well for solids [20]. As it involves the power of the density matrix we refer to it as "power functional" in the following. The power $\alpha$ lies in the interval $1 / 2 \leq \alpha \leq 1$. In the limit $\alpha=1$, minimization of the total-energy functional (1) yields the Hartree-Fock energy, while the case $\alpha=1 / 2$ corresponds to the Müller functional which tends to overcorrelate $[3,11]$. Hence the power $\alpha$ plays a similar role as the mixing parameter in the hybrid functionals of the DFT.

The situation as it stands is that most of the existing $\mathrm{xc}$ functionals of RDMFT are designed for finite systems and perform quite poorly for solids and the HEG. The power functional, on the other hand, is designed for the case of solids, but has not yet been applied to the HEG or finite systems. The objective of the present work is to fill this gap, in the pursuit of a functional form that works equally well for both finite and extended systems.

It is difficult to overstate the importance of the HEG in the development of many-body theories. It is not only used as a benchmark, but also acts as a reference system for the design of $\mathrm{xc}$ functionals. Within DFT, the LDA is perhaps the most successful example of this. Furthermore, results for the HEG give us valuable indications on how the theory will perform especially for metallic systems.

With this in mind, we first compare the relative performance of various RDMFT functionals in reproducing the correlation energy of the HEG of various densities. Figure 1 is a plot of the correlation energy versus the density parameter $r_{s}$ for a variety of approximate functionals compared to exact Monte Carlo values [21,22]. Clearly, the Müller [23], corrected Hartree-Fock (CHF) [10], and Csányi-GoedeckerArias (CGA) [12] functionals perform poorly over the whole range of $r_{s}$. The BBC [13] and PNOF [14] functionals are more reasonable but still far from the true result. Encouragingly, we find that for $\alpha$ between 0.55 and 0.58 , the power functional lies very close to the Monte Carlo results and possesses a good low-density limit, making it one of the best 1-RDM functionals for the HEG.

Since the power functional performs well for the HEG at small values of $r_{s}$ and for periodic solids [20], it is worthwhile to investigate its behavior for finite systems. First we performed a free optimization of the parameter $\alpha$ using a set of 54 molecules and radicals. These molecules form a subset of the Gaussian-2 (G2) test set of molecules [24,25]. For this optimization two different basis sets are employed, namely,

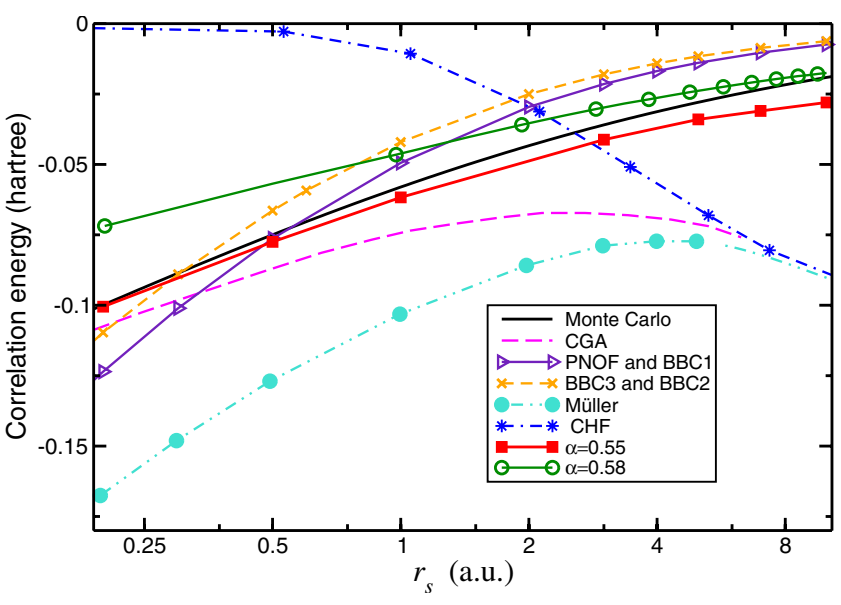

FIG. 1. (Color online) Correlation energy as a function of the Wigner-Seitz radius for the homogeneous electron gas. RDMFT results are obtained using various approximations to the $\mathrm{xc}$ functional. Monte Carlo results are taken from Ref. [21] (see also Ref. [22]).

6-31G* and cc-pVDZ. The optimal value of $\alpha$ that minimizes the error in the correlation energy for this set of molecules is 0.578. This value is essentially the same for both kinds of basis set used in the present work. It is interesting to note from Fig. 1 that the value of $\alpha$ which best reproduces the Monte Carlo data for $r_{s}$ of interest for most atoms and molecules is about 0.55 . The optimal value obtained for the set of molecules, $\alpha=0.578$, is quite close to this.

Having determined the optimal value for the parameter $\alpha$, we compare this approach with different RDMFT functionals. Figure 2 is the plot of relative error in the correlation energy $(\delta)$ given by

$$
\delta=100 \sqrt{\sum\left[\left(E_{c}-E_{c}^{(\mathrm{ref})}\right) / E_{c}^{(\mathrm{ref})}\right]^{2} / N_{\mathrm{mol}}},
$$

where $E_{c}$ is the correlation energy obtained with the method under consideration, $E_{c}^{(\mathrm{ref})}$ is the reference correlation energy

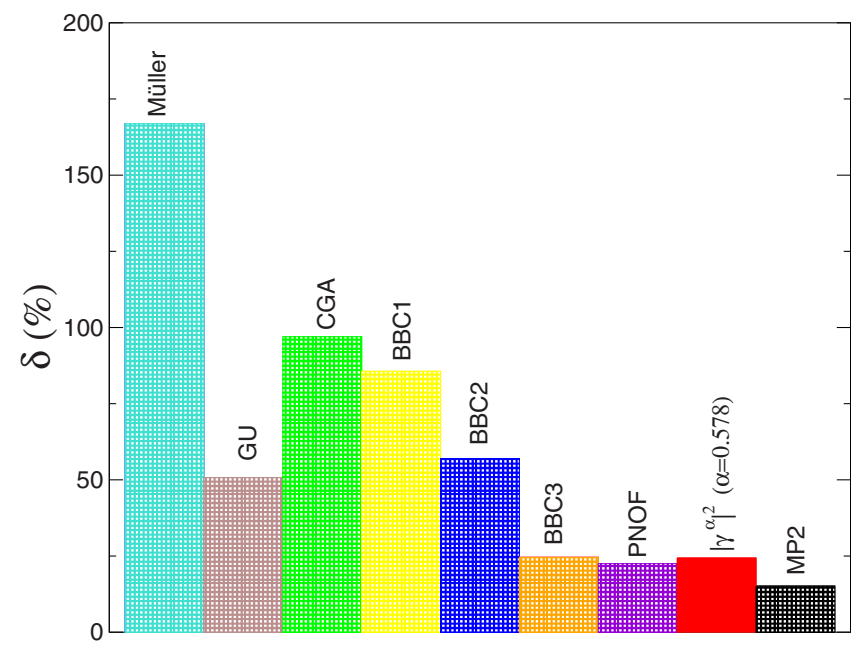

FIG. 2. (Color online) Percentage deviation of the correlation energy, obtained using various 1-RDM functionals, from the exact $\operatorname{CCSD}(\mathrm{T})$ results. 


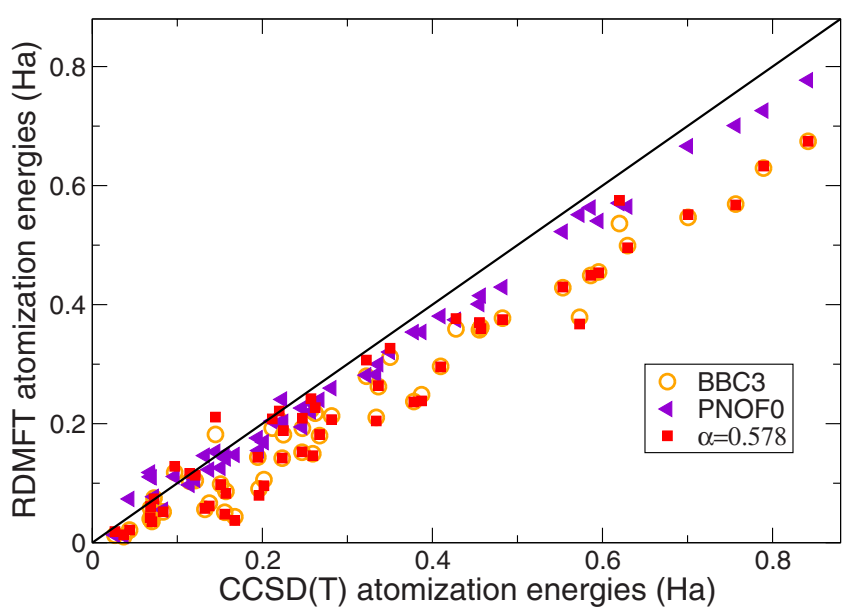

FIG. 3. (Color online) Atomization energies for the G2 set of molecules calculated using the $\mathrm{BBC} 3$, PNOF0, and power functional vs the $\operatorname{CCSD}(\mathrm{T})$ reference atomization energies.

which is obtained with the $\operatorname{CCSD}(\mathrm{T})$ method, and $N_{\text {mol }}$ is the number of systems included in the calculation. It is apparent from Fig. 2 that the power functional is very good in determining the correlation energy of finite systems. In fact, we find that this very simple functional with $\alpha=0.578$ (same value of $\alpha$ was used for the full G2 set) is of similar quality as the $\mathrm{BBC} 3$ or the PNOF0 which are to date the best RDMFT functionals as far as finite systems are concerned $[3,14,16-19]$.

The accurate determination of atomization energies is important for the calculation of the energetics of any chemical reaction. Hence this important quantity also acts as a test for the quality of an approximate functional. In Fig. 3 we plot the atomization energies of molecules of the entire G2 set obtained with various approximate functionals within RDMFT versus the reference value determined using the $\operatorname{CCSD}(\mathrm{T})$ method.

It is clear from Ref. [3] and Fig. 2 that BBC3, PNOF0, and the power functional are the most accurate among the $\mathrm{xc}$ functionals we considered; hence, in Fig. 3, we only compare these three functionals. It is clearly visible that the power functional is comparable in accuracy to the $\mathrm{BBC} 3$ functional, while PNOF0 is slightly better than the two.

The successful prediction of properties of molecules at equilibrium does not necessarily imply a good performance for strongly distorted molecules, with the dissociation limit being one such example. For a stretched molecule, not only the total energy has to be equal to the sum of the energies of the fragments but also the occupations of the natural orbitals have to be correct. The simplest example is perhaps the $\mathrm{H}_{2}$ molecule. If we take the hydrogen atoms far apart, the total energy should go to -1 a.u. and the four occupied spin orbitals made from the hydrogen $1 s$ states have to be occupied by half an electron each. Many DFT and RDMFT functionals fail to reproduce the correct dissociation of this system $[26,27]$.

In Fig. 4, we plot the $\mathrm{H}_{2}$ dissociation curve obtained using various 1-RDM functionals, together with the exact curve obtained through a full configuration-interaction calculation.

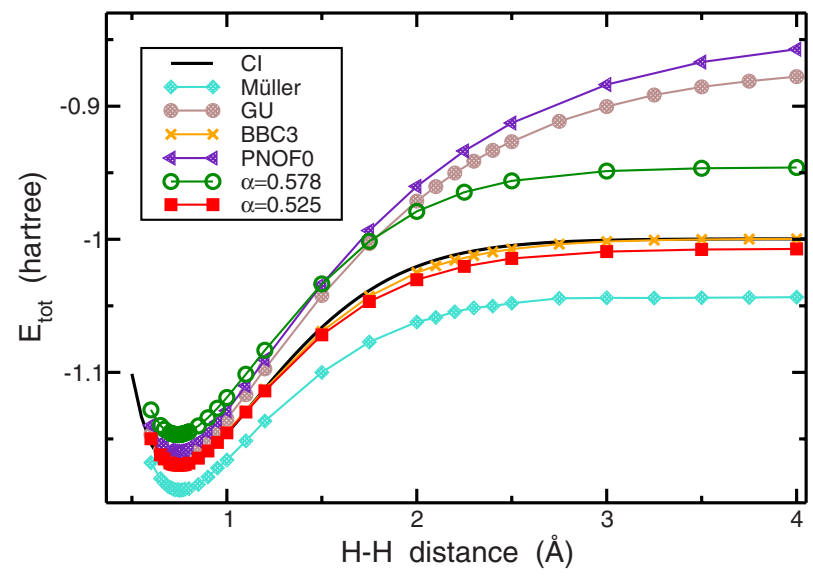

FIG. 4. (Color online) Total energy of the $\mathrm{H}_{2}$ molecule (in a.u.) vs the distance (in $\AA$ ) between the two hydrogen atoms. RDMFT results are obtained using various approximations to the $\mathrm{xc}$ functional. For reference the configuration interaction results are also presented (black line).

The BBC3 functional is designed with the dissociation limit in mind and it yields the most accurate results for the $\mathrm{H}_{2}$ dissociation. The failure of PNOF0 and GU functionals is twofold: as the distance between the two $\mathrm{H}$ atoms increases, the energy deviates strongly from the exact value. Second, at a sufficiently large distance between the two $\mathrm{H}$ atoms $(6 \AA)$ two of the bonding orbitals still have occupancy of 0.86 and the other two have 0.14. Both the Müller and the power functionals with $\alpha=0.578$ give the correct occupancy of $\sim 0.5$ for all four bonding spin orbitals, but the dissociation energy is underestimated by the former of these functionals and overestimated by the latter. If, on the other hand, the value of $\alpha$ is changed to 0.525 the power functional describes the $\mathrm{H}_{2}$ dissociation curve accurately, with an accuracy comparable to the $\mathrm{BBC} 3$ functional.

In all the examples studied, it is clear that the simple form of the power functional suffices to obtain very good electronic properties. However, we are faced with a problem: the optimal value for $\alpha$ varies from one kind of system to another. In fact, we find $\alpha=0.65$ for solids, $\alpha=0.55$ for the HEG, $\alpha=0.578$ for molecules at equilibrium, and $\alpha=0.525$ for stretched $\mathrm{H}_{2}$. Although one can use different values of $\alpha$ for different materials, it would be desirable to have a unique method to determine the system-dependent value of $\alpha$. In this regard, one could make $\alpha$ itself a functional of the $1-R D M$ and optimize it in as ab initio manner for each system. Many other improvements of the power functional are also conceivable; for example, it could be a basis for sophisticated corrections like those of Gritsenko et al. [13] and/or removal of self-interaction terms. How these modifications affect the quality of the power functional will be the subject of future studies.

In summary, we have used the recently proposed power functional to calculate the correlation energy of the HEG, G2 test set of molecules, and stretched $\mathrm{H}_{2}$. For the case of molecules, we also determined atomization energies. Our results show that the power functional, originally proposed for 
solids, also performs very well for the HEG and finite systems. However, the optimal value of $\alpha$ for all three cases is different. The encouraging results of the present work point to the fact that this family of approximations is an interesting path for the future development of approaches, within RDMFT, to accurately describe electronic correlations.
This work was supported by the Deutsche Forschungsgemeinshaft (Contract No. SPP 1145) and by the EC Network of Excellence NANOQUANTA (Contract No. NMP4-CT2004-500198). Part of the calculations was performed at the Laboratório de Computação Avançada of the University of Coimbra.
[1] A. D. Becke, J. Chem. Phys. 98, 5648 (1993).

[2] C. Lee, W. Yang, and R. G. Parr, Phys. Rev. B 37, 785 (1988).

[3] N. N. Lathiotakis and M. A. L. Marques, J. Chem. Phys. 128, 184103 (2008).

[4] P.-O. Löwdin, Phys. Rev. 97, 1474 (1955).

[5] T. L. Gilbert, Phys. Rev. B 12, 2111 (1975).

[6] C. Kollmar, J. Chem. Phys. 121, 11581 (2004).

[7] J. Cioslowski, K. Pernal, and M. Buchowiecki, J. Chem. Phys. 119, 6443 (2003).

[8] K. Pernal and J. Cioslowski, J. Chem. Phys. 120, 5987 (2004).

[9] J. Cioslowski and K. Pernal, Phys. Rev. B 71, 113103 (2005).

[10] G. Csányi and T. A. Arias, Phys. Rev. B 61, 7348 (2000).

[11] N. N. Lathiotakis, N. Helbig, and E. K. U. Gross, Phys. Rev. B 75, 195120 (2007).

[12] G. Csányi, S. Goedecker, and T. A. Arias, Phys. Rev. A 65 , 032510 (2002).

[13] O. Gritsenko, K. Pernal, and E. J. Baerends, J. Chem. Phys. 122, 204102 (2005).

[14] M. Piris, Int. J. Quantum Chem. 106, 1093 (2006).

[15] M. A. L. Marques and N. N. Lathiotakis, Phys. Rev. A 77, 032509 (2008).

[16] P. Leiva and M. Piris, Int. J. Quantum Chem. 107, 1 (2007).
[17] P. Leiva and M. Piris, J. Theor. Comput. Chem. 4, 1165 (2005).

[18] P. Leiva and M. Piris, J. Chem. Phys. 123, 214102 (2005).

[19] K. Pernal and J. Cioslowski, Chem. Phys. Lett. 412, 71 (2005).

[20] S. Sharma, J. K. Dewhurst, N. N. Lathiotakis, and E. K. U. Gross, Phys. Rev. B 78, 201103(R) (2008).

[21] G. Ortiz and P. Ballone, Phys. Rev. B 56, 9970 (1997).

[22] In Ref. [21] the correlation energy of the three-dimensional uniform electron gas is calculated using variational Monte Carlo and fixed-node diffusion Monte Carlo methods. The accuracy of the extrapolated correlation energy is estimated to be 4 mhartree.

[23] A. M. K. Müller, Phys. Lett. 105A, 446 (1984).

[24] L. A. Curtiss, K. Raghavachari, P. C. Redfern, and J. A. Pople, J. Chem. Phys. 106, 1063 (1997).

[25] L. A. Curtiss, P. C. Redfern, K. Raghavachari, and J. A. Pople, J. Chem. Phys. 109, 42 (1998).

[26] E. J. Baerends, Phys. Rev. Lett. 87, 133004 (2001).

[27] O. Gritsenko and E. J. Baerends, Int. J. Quantum Chem. 106, 3167 (2006). 